\title{
Fuzzy Cognitive Maps-Based Method for Modelling Tacit Knowledge of Scheme Evaluation
}

\author{
Zhongming Chen ${ }^{1, *}$, Yan Yan $^{1}$, Jia Hao ${ }^{1}$ and Guoxin Wang ${ }^{1}$ \\ ${ }^{1}$ School of Mechanical Engineering, Beijing Institute of Technology, Beijing, China
}

\begin{abstract}
The design scheme evaluation is an important part of product intelligent design system, but the existing scheme evaluation methods have not considered the problem of the mutual influence of the evaluation index, thus reducing the objectivity of the evaluation result. In fact, the influence relationship of the scheme evaluation index is a kind of tacit knowledge which exists in the expert's mind. Therefore, this paper introduces the fuzzy cognitive maps, which is a tacit knowledge expression model, to model the influence relationship among the evaluation indexes. This modelling process has two interconnected parts: the modelling process of domain variables and the modelling process of causal structure. And this modelling process has the meaning of guiding the practical operation, and laying the foundation for further creating a more optimized scheme evaluation method.
\end{abstract}

\section{Introduction}

Product intelligent design system provides possibility for automatically and intelligently designing product, and the evaluation and decision-making of product design schemes is an important part of this system. Typically, designers input design requirements, then the system will output a variety of alternatives by its reasoning module. However, designers often want to get a unique optimal scheme for their subsequent research and production. Therefore, it needs an evaluation and optimization module to evaluate and rank these alternatives, and finally gets the optimal scheme[1].

However, the existing scheme evaluation methods have not considered the problem of the influence relationship among the evaluation indexes, thus reducing the objectivity of the evaluation result. The reason is usually that it is unable to obtain the influence relationship among the evaluation indexes.

But in fact, because the scheme evaluation is done by the evaluation personnel, and the evaluation indexes are also formulated by the domain experts, at the same time, the influence relationship among the evaluation indexes have some common features with the tacit knowledge, such as difficult to understand, difficult to use digital or formula to express, not yet been dominant and so on. Therefore, the influence relationship among the scheme evaluation indexes is a kind of tacit knowledge which exists in the expert's mind. If the modelling and expression method of tacit knowledge is introduced into the field of scheme evaluation, it is bound to be an effective way to solve the problem.

Based on this kind of consideration, this paper introduces the fuzzy cognitive maps, which is a tacit knowledge expression model, to model the influence relationship among the evaluation indexes.

The remainder of the paper is as follows: in Section 2, we discuss important literature related to the modelling and expression methods of tacit knowledge, and determine the fuzzy cognitive maps as the final method. In Section 3, we propose the modelling process of the influence relationship among the evaluation indexes based on fuzzy cognitive maps, and the modelling process has two interconnected parts: the modelling process of domain variables and the modelling process of causal structure. In Section 4, we conclude the paper and indicate future research issues.

\section{Method choice}

There are many different methods to model and express the tacit knowledge, and from different point of view, there will be different emphases. For example, management science emphasizes the knowledge transformation, and focuses on how to build a scientific knowledge management model in order to facilitate knowledge sharing. While the research in engineering field is usually to seek the method of tacit knowledge dominance. The main methods are ontology, cognitive maps, fuzzy cognitive maps, and knowledge map etc.

Ontology, as a relatively mature specification format of knowledge expression, plays an important role in the tacit knowledge expression and construction. A lot of methods about tacit knowledge expression are based on ontology. Such as Chen proposed a method for ontologybased empirical knowledge representation and reasoning[2], and Sun developed an ontology-based approach to support knowledge-intensive product design[3]. Although these studies have made a lot of contributions to the application of ontology in the field of tacit knowledge, but the ontology is weak in meeting the needs of tacit knowledge visualization.

Cognitive maps is a human brain cognitive model proposed by American psychologist Tolman, which is widely used in knowledge mining, personal knowledge management, and knowledge sharing[4]. However, only limited information can be expressed by the cognitive maps model, such as it cannot quantitatively express the causal relationship among knowledge nodes.

Fuzzy cognitive maps was proposed by Kosko in 1986, which is a dynamic modelling method for knowledge representation and causal reasoning[5]. It is easy to use, easy to understand, and can be used for 
quantitative causal inference to test the changes of the system. Compared to the cognitive maps, fuzzy cognitive maps introduces the fuzzy theory, so it has more advantages in reasoning, prediction and the quantitative expression of the causal relationship among the knowledge nodes.

Knowledge map is a kind of integrated logical view of various kinds of resources, which can be effectively provided to the users for knowledge inquiry. But in essence, it is a kind of knowledge index, and there is no deep discussion on the modelling and expression of tacit knowledge. Therefore, the knowledge map has a disadvantage in computation and reasoning.

Because the tacit knowledge modelling and expression method required by this research is to be applied in the field of scheme evaluation, and it is necessary to have advantages in quantitative expression and reasoning. Therefore, the fuzzy cognitive maps is the most suitable method.

\section{Modelling process}

Fuzzy cognitive maps model has two basic elements: domain variables and causal structure. The domain variables are the knowledge nodes of the current task, and the causal structure is the relationship among the knowledge nodes. In the view of influence relationship among the evaluation indexes, the domain variable refers to the evaluation index of the scheme evaluation index system, and the causal structure is the influence relationship among the evaluation indexes. Then, we discuss the method of modelling the domain variables and the causal structure.

\subsection{Modelling process of domain variables}

Generally speaking, modelling domain variables in fuzzy cognitive maps usually uses heuristic questions, which are unstructured, exploratory and open. In the concrete operation process, first, determine the highest goal, on behalf of the tacit knowledge that we finally want to get. And the highest goal is the starting point for the process of questioning. Then, inspired by heuristic questions, the experts are required to list all the relevant knowledge nodes about the highest goal as the first level domain variables. Subsequently, for each of the first level domain variable, the experts then list all the relevant knowledge nodes as the second domain variables. Repeat the above process, build the third level domain variables or more, until the experts believe that there is no more domain variables about the highest goal.
The above is a general method to model the domain variables of the fuzzy cognitive maps, but it needs to be adjusted according to the specific field of the scheme evaluation, because a scheme evaluation problem usually has a specific background. That is, the evaluation index system is a very professional question, which needs professional discussion of domain experts, such as building the guiding principles of the index system, carefully removing or selecting the index according to the guiding principles and actual situation, and finally selecting a number of important evaluation indexes constituting the scientific evaluation index system. Therefore, the general method to model the domain variables of the fuzzy cognitive maps can only be used as the first stage of the establishment of evaluation index system, and the second stage needs to add the professional discussion.

Therefore, this paper builds the domain variables modelling process of the scheme evaluation index based on the fuzzy cognitive maps, as shown in Figure 1.

First, determine the highest goal, such as "the factors that need to be considered about a good XX scheme".

Second, the experts are required to list all the relevant influence factors about the highest goal as the first level domain variables, inspired by heuristic questions, such as "What factors do you think have a direct influence on choosing a good XX scheme?" Subsequently, for each of the first level domain variable, the experts then list all the relevant influence factors as the second domain variables, such as "So, for the factor XXX, what other factors do you think have a direct influence on it?" Repeat the above process, build the third level domain variables or more, until the experts believe that there is no more influence factors about the highest goal.

The third step is to determine the final scheme evaluation index system by the domain experts. The process can be started with building guiding principles of the index system. And then carefully remove or select the index according to the guiding principles and actual situation. Finally select a number of important evaluation indexes constituting the evaluation index system.

At last, every evaluation index in the scheme evaluation index system is the domain variable that can be applied to the subsequent causal structure modelling.

\subsection{Modelling process of causal structure}

Through the modelling process of domain variables, the evaluation index system is determined. On this basis, the causal structure can be modelled, i.e. the influence relationship among the evaluation indexes can be obtained. 


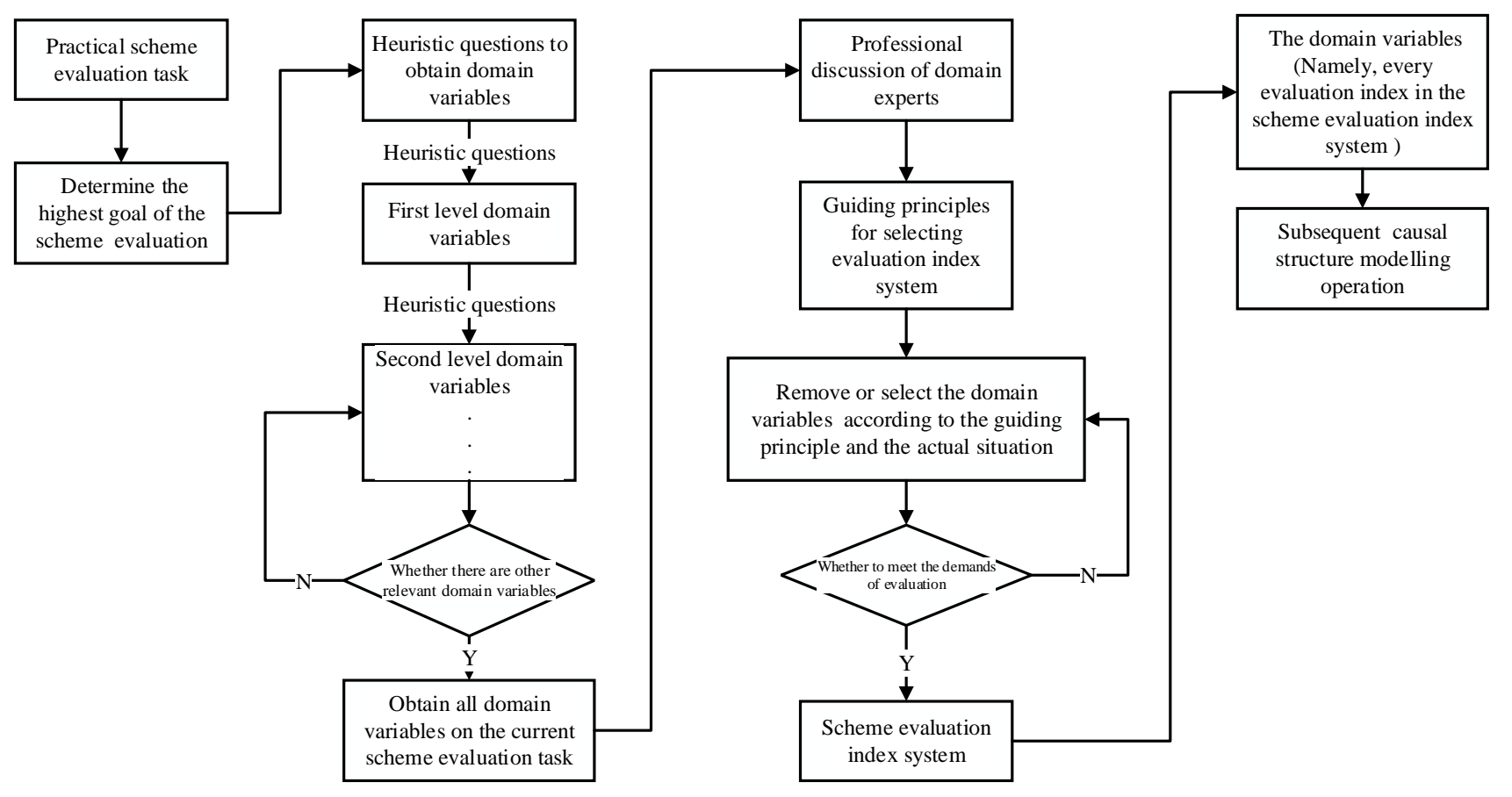

Figure 1. The domain variables modelling process

There are different types of the influence relationship, positive correlation, negative correlation or not related. And every influence relationship needs to be given the influence relationship strength. Due to it is easier for experts to understand discrete linguistic strength value than continuous numerical influence strength value. Therefore, the linguistic influence strength table is established firstly. The linguistic influence strength table contains five kinds of influence strength values, namely: "very strong, strong, medium, weak, and very weak".

In the actual modelling process of causal structure, the experts will be required to determine whether there is influence relationship between each of the two domain variables. If they influence each other, then determine whether a positive correlation or negative correlation, and choose a reasonable influence strength value from the linguistic influence strength table; if there is no relationship between the two domain variables, then they are not relevant.

In particular, in the judgment of the influence relationship, the influence of A on B is different from B on A. Taking "precision" and "economic cost" as an example. Improving "precision" will inevitably lead to the increase of "economic costs", and the degree is great. Therefore, the influence of "precision" on "economic cost" is "positive correlation", the linguistic influence strength value is "very strong". But in turn, the increase of "economic costs" does not necessarily lead to the increase of "precision", so it can only say that the influence of "economic cost "on" precision" is not relevant. Therefore, if there are " $n$ " domain variables, that is, there are " $n$ " evaluation indexes in the evaluation index system, then the determining of influence relationship strength should be executed "n(n-1)" times, rather than "n(n-1)/2" times.

Applying linguistic influence strength is only for the convenience of experts understanding. But the causal structure needs to be quantitative expressed in the fuzzy cognitive maps. Therefore, after the linguistic influence strength is obtained, it needs to be converted into a numerical type. The numerical influence strength type has two advantages, which can quantitatively express the causal structure and support the inference calculation. The numerical influence strength usually uses $\left[\begin{array}{llll}-1 \ldots & 0 . . & 1\end{array}\right]$ figures to express. Hence, it needs to establish a corresponding table between the linguistic influence strength and the numerical influence strength, as shown in Table 1.

Table 1. The corresponding table between the linguistic influence strength and the numerical influence strength.

\begin{tabular}{|c|c|c|}
\hline Influence type & $\begin{array}{c}\text { Linguistic } \\
\text { influence strength }\end{array}$ & $\begin{array}{c}\text { Numerical } \\
\text { influence strength }\end{array}$ \\
\hline \multirow{4}{*}{$\begin{array}{c}\text { Positive } \\
\text { correlation }\end{array}$} & very strong & 1.0 or 0.9 \\
\cline { 2 - 3 } & strong & 0.8 or 0.7 \\
\cline { 2 - 3 } & medium & 0.6 or 0.5 \\
\cline { 2 - 3 } & weak & 0.4 or 0.3 \\
\cline { 2 - 3 } & very weak & 0.2 or 0.1 \\
\hline Not related & not related & 0 \\
\hline \multirow{3}{*}{$\begin{array}{c}\text { Negative } \\
\text { correlation }\end{array}$} & very strong & -1.0 or -0.9 \\
\cline { 2 - 3 } & strong & -0.8 or -0.7 \\
\cline { 2 - 3 } & medium & -0.6 or -0.5 \\
\cline { 2 - 3 } & weak & -0.4 or -0.3 \\
\cline { 2 - 3 } & very weak & -0.2 or -0.1 \\
\hline
\end{tabular}

Therefore, the modelling process of causal structure can be summarized as shown in Figure 2 .

First, the experts determine whether there is influence relationship between each of the two domain variables. If they influence each other, then determine whether a positive correlation or negative correlation, and from the linguistic influence strength table to choose a reasonable influence strength value; if there is no relationship between the two domain variables, then they are not relevant. And then in the help of the corresponding table 


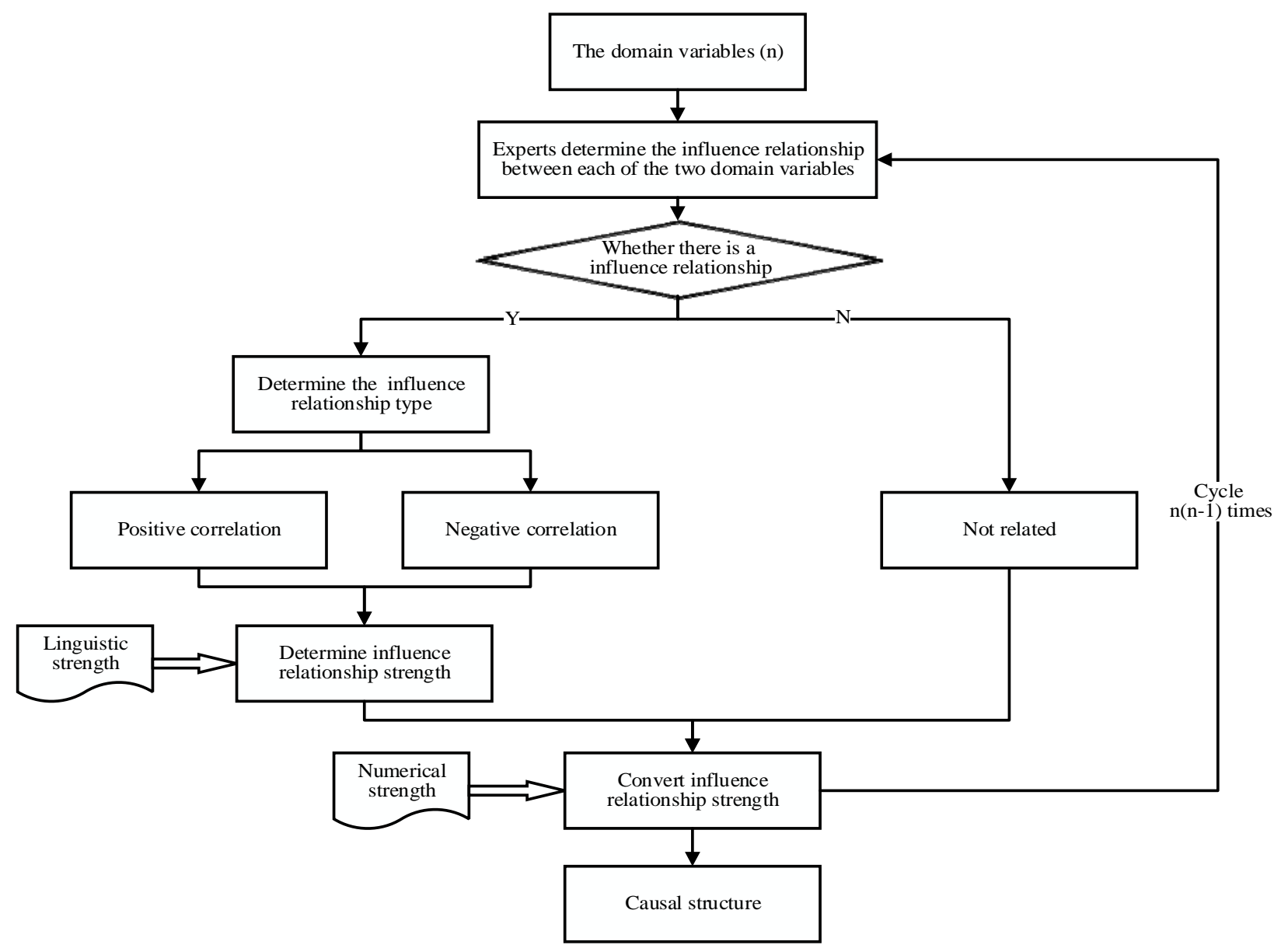

Figure 2. The causal structure modelling process

between the linguistic influence strength and the numerical influence strength, the linguistic influence strength is converted to numerical influence strength. The above process repeats $n(n-1)$ times to ensure that any two domain variables have been compared, and finally get the causal structure.

\section{Conclusion}

To solve the problem of influence relationship among the evaluation indexes, this paper introduces tacit knowledge to scheme evaluation field, and applies fuzzy cognitive maps method to propose a modelling process of the influence relationship among the evaluation indexes. This modelling process has two interconnected parts: the modelling process of domain variables and the modelling process of causal structure. And this modelling process has the meaning of guiding the practical operation, and laying the foundation for creating a more optimized scheme evaluation method. Therefore, the next step of the research is to realize the integration of the tacit knowledge model and the existing scheme evaluation method.

\section{Acknowledgement}

Supported by the National Natural Science Foundation of China (No. 51505032 and No. 51375049), and Ministries project (No. JSJC201402).

\section{References}

[1] H.W. Wang, G. Liu, Z.D. Yang, J. Harbin Inst. Technol. 46, 99 (2014)

[2] Y.J. Chen, Decis. Sup. S. 50, 1(2010)

[3] W. Sun, Q.Y. Ma, T.Y. Gao, S. Chen, Int. J. Adv. M. 48,421(2010)

[4] I. Clarke. J. Mgm. Stud. 38,147(2001)

[5] B. Kosko. Int. J. Med. Stud. 24,65(1986) 